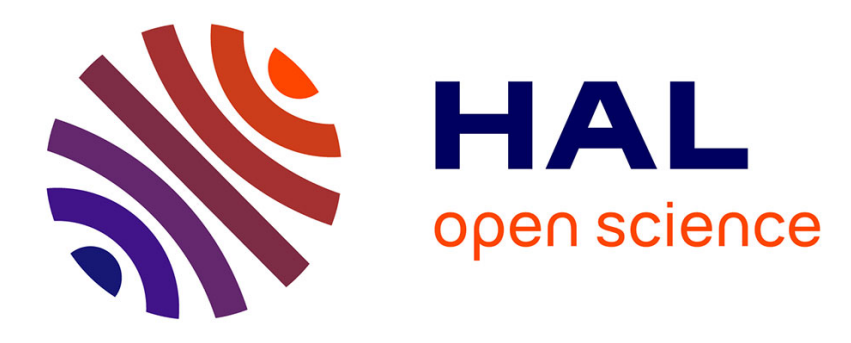

\title{
Electron screening corrections in dense ionized matter
}

\author{
J.P. Hansen
}

\section{To cite this version:}

J.P. Hansen. Electron screening corrections in dense ionized matter. Journal de Physique Lettres, 1975, 36 (5), pp.133-135. 10.1051/jphyslet:01975003605013300 . jpa-00231171

\section{HAL Id: jpa-00231171 https://hal.science/jpa-00231171}

Submitted on 1 Jan 1975

HAL is a multi-disciplinary open access archive for the deposit and dissemination of scientific research documents, whether they are published or not. The documents may come from teaching and research institutions in France or abroad, or from public or private research centers.
L'archive ouverte pluridisciplinaire HAL, est destinée au dépôt et à la diffusion de documents scientifiques de niveau recherche, publiés ou non, émanant des établissements d'enseignement et de recherche français ou étrangers, des laboratoires publics ou privés. 


\title{
ELECTRON SCREENING CORRECTIONS IN DENSE IONIZED MATTER
}

\author{
J. P. HANSEN \\ Laboratoire de Physique Théorique des Liquides (*) \\ Université Pierre-et-Marie-Curie, 4, place Jussieu, 75005 Paris, France
}

(Reçu le 3 février 1975, accepté le 6 mars 1975)

\begin{abstract}
Résumé. - A l'aide de la théorie des perturbations thermodynamiques nous avons calculé les corrections à l'énergie libre et à l'équation d'état du plasma à une composante, dues à la polarisation des électrons formant le fond continu.
\end{abstract}

\begin{abstract}
Thermodynamic perturbation theory is used to calculate the corrections to the free energy and equation of state of the one-component plasma, due to the polarization of the electron background.
\end{abstract}

In a series of recent papers $[1,2,3]$ the thermodynamic properties and correlation functions of the classical one-component plasma (OCP) in a uniform background have been computed accurately with the help of computer simulation techniques. The OCP is a reasonable model for the description of superdense, completely ionized matter typical of extreme astrophysical situations (e.g. white dwarfs and the interior of Jupiter) and possibly of metallic hydrogen. Under such conditions, the electrons are strongly degenerate and in the first approximation play the role of a rigid, uniform background.

However, even a highly degenerate electron gas will be polarized by the ionic charge distribution; consequently the ion-ion interaction will be modified by the electron screening. Hubbard and Slattery [4] have shown how this effect can be accounted for through the dielectric function of the electron gas. In this letter electron screening corrections will be calculated with the help of thermodynamic perturbation theory. These screening corrections will be included in a simple equation of state for dense ionized matter. It should be noted that the electron screening lenght breaks the scale invariance of the OCP by which all equilibrium properties depend only on the single dimension less parameter

$$
\Gamma=\frac{\beta(Z e)^{2}}{a},
$$

where $Z e$ is the charge of the ions, $a=\left(\frac{4 \pi \rho}{3}\right)^{-1 / 3}$

$\left(^{*}\right)$ Equipe associée au Centre National de la Recherche Scientifique (C.N.R.S.). is the ion-sphere radius $(\rho=N / V$ is the number density) and $\beta=1 / k_{\mathrm{B}} T$.

Because of the large mass ratio between ions and electrons, the latter react practically instantanously to any variation of the ion charge density which plays the role of an external charge density :

$$
\rho_{\mathrm{ext}}(\mathbf{r})=Z e \sum_{i=1}^{N} \delta\left(\mathbf{r}-\mathbf{r}_{i}\right)
$$

where the $\mathbf{r}_{i}(1 \leqslant i \leqslant N)$ are the ion positions and $\rho_{\text {ext }}(\mathbf{r})$ has the following Fourier transform :

$$
\tilde{\rho}_{\mathrm{ext}}(\mathbf{k})=\frac{Z e}{V} \sum_{i=1}^{N} \mathrm{e}^{i \mathbf{k} \cdot \mathbf{r}_{i}} .
$$

Hence the induced charge density can be related to the external charge density through the stratic dielectric function $\varepsilon(\mathbf{k})$ of the electron gas $[2,4,5]$ :

$$
\tilde{\rho}_{\text {ind }}(\mathbf{k})=\left[\frac{1}{\varepsilon(\mathbf{k})}-1\right] \tilde{\rho}_{\text {ext }}(\mathbf{k})
$$

From Poisson's equation the corresponding total electrostatic potential is :

$$
\begin{aligned}
\tilde{\varphi}(\mathbf{k}) & =\frac{4 \pi Z e}{k^{2} \varepsilon(\mathbf{k})} \tilde{\rho}_{\text {ext }}(\mathbf{k})= \\
& =\frac{4 \pi Z e}{k^{2}} \tilde{\rho}_{\text {ext }}(\mathbf{k})+\frac{4 \pi Z e}{k^{2}}\left[\frac{1}{\varepsilon(\mathbf{k})}-1\right] \tilde{\rho}_{\text {ext }}(\mathbf{k})
\end{aligned}
$$

where the first term stems from the external charge density and the second term from the induced charge density. Replacing $\rho_{\text {ext }}(\mathbf{k})$ by $\rho_{\mathbf{k}}$ for brevity, 
the Hamiltonian of the system then reads :

$$
\begin{aligned}
\mathcal{H}=\frac{1}{2 V} & \sum_{\mathbf{k}}^{\prime} \frac{4 \pi(Z e)^{2}}{k^{2}}\left(\rho_{\mathbf{k}} \rho_{-\mathbf{k}}-N\right)+ \\
& +\frac{1}{2 V} \sum_{\mathbf{k}}^{\prime} \frac{4 \pi(Z e)^{2}}{k^{2}}\left[\frac{1}{\varepsilon(\mathbf{k})}-1\right] \rho_{\mathbf{k}} \rho_{-\mathbf{k}} .
\end{aligned}
$$

The prime denotes that the term $\mathbf{k}=0$ is omitted from the sum, due to the uniform (non polarized) background. In the first term $N$ is substracted from $\rho_{\mathbf{k}} \rho_{-\mathbf{k}}$ in order to eliminate the infinite self-energy of the ions. The first term in (4), called the direct ionion interaction, is identical with the Hamiltonian of the unscreened OCP ; the second term corresponds to the indirect, or electron-induced, ion-ion interaction, which vanishes in the limit of zero screening $(\varepsilon(\mathbf{k})=1)$. (4) is easily recast into the from :

$$
\mathcal{H}=\frac{1}{2 V} \sum_{\mathbf{k}}^{\prime} \frac{4 \pi(Z e)^{2}}{k^{2} \varepsilon(\mathbf{k})}\left(\rho_{\mathbf{k}} \rho_{-\mathbf{k}}-N\right)+U_{0}
$$

where :

$$
U_{0}=\frac{N}{2 V} \sum_{\mathbf{k}}^{\prime} \frac{4 \pi(Z e)^{2}}{k^{2}}\left[\frac{1}{\varepsilon(\mathbf{k})}-1\right]
$$

is a structure-independent constant. In r-space the full ion-ion potential is now given by the Fourier transform :

$$
v(r)=\frac{1}{V} \sum_{\mathbf{k}} \frac{4 \pi(Z e)^{2}}{k^{2} \varepsilon(\mathbf{k})} \mathrm{e}^{i \mathbf{k} . \mathbf{r}}=\frac{1}{V} \sum_{\mathbf{k}} \tilde{v}(\mathbf{k}) \mathrm{e}^{i \mathbf{k} . \mathbf{r}}
$$

which reduces to $(Z e)^{2} / r$ in the OCP limit.

If the electron density $Z \rho$ is characterized by the usual dimensionless parameter $r_{\mathrm{s}}=\left(3 / 4 \pi Z \rho a_{0}^{3}\right)^{1 / 3}$ ( $a_{0}$ is the electronic Bohr radius), normal white dwarf conditions (i.e. a density of $10^{6} \mathrm{~g} / \mathrm{cm}^{3}$ and a predominantly He composition) correspond to $r_{\mathrm{s}} \simeq 0.015$, whereas the Jovian interior (density $\simeq 30 \mathrm{~g} / \mathrm{cm}^{3}$ and a predominantly $\mathscr{H}$ composition) would correspond to $r_{\mathrm{s}} \simeq 0.4$. These figures are respectively two and one orders of magnitude below the $r_{\mathrm{s}}$ values at metallic densities. The electron screening is therefore expected to be considerably weaker under the extreme astrophysical conditions than in the usual metals. Hence it seems reasonable to treat the effect of electron screening as a correction to the OCP within the framework of thermodynamic perturbation theory [6]. In fact perturbation theory has already been used to treat the screened Coulomb gas [7], but taking a hard sphere fluid as the reference (unperturbed) system. Such a procedure is expected to give good results in the strong screening limit, whereas the choice of the OCP as a reference system naturally seems more appropriate in the weak screening limit, which is precisely considered in this work. There remains the problem of choosing the dielectric function $\varepsilon(\mathbf{k})$ of the electron gas. In the density range $r_{\mathrm{s}} \lesssim 1$, the Lindhard dielectric function $[5,8]$ obtained in the framework of the random phase approximation (RPA), can be expected to be adequate. However, in order to illustrate the perturbation theory which we propose and to obtain the simplest possible expressions, in this paper we choose to replace the Lindhard dielectric function by its small $k$ limit, which reduces to the Thomas-Fermi approximation [5] :

$$
\varepsilon(k)=1+k_{\mathrm{TF}}^{2} / k^{2}
$$

where $k_{\mathrm{TF}}=(12 / \pi)^{1 / 3} /\left(a_{0} r_{\mathrm{s}}^{1 / 2}\right)$ is the inverse of the Thomas-Fermi screening length. In a subsequent paper we shall present results obtained with more realistic dielectric functions, in order to allow quantitative comparisons with the results of references [4] and [8].

To calculate the Helmholtz free energy of the screened ionic system, we start from the familiar exact expression [9] :

$$
\begin{aligned}
\frac{\beta F}{N}=\frac{\beta F^{(0)}}{N}+ & \frac{\beta U_{0}}{N}+\frac{1}{2(2 \pi)^{3}} \int_{0}^{1} \times \\
& \times \mathrm{d} \lambda \int[S(k ; \lambda)-1] \tilde{w}(k) \mathrm{d}^{3} k
\end{aligned}
$$

where the superscript ${ }^{(0)}$ will henceforth refer systematically to the reference system (the OCP),

$$
\widetilde{w}(k)=\beta\left[\widetilde{v}(k)-\widetilde{v}^{(0)}(k)\right]=-\frac{4 \pi \beta(Z e)^{2} k_{\mathrm{TF}}^{2}}{k^{2}\left(k^{2}+k_{\mathrm{TF}}^{2}\right)}
$$

is the perturbation part of the potential and $S(k ; \lambda)$ is the structure factor for a system of ions interacting through the potential $\widetilde{v}^{(0)}(k)+\lambda \widetilde{w}(k)$;

$$
S(k ; 0) \equiv S^{(0)}(k) \text {. }
$$

Adding and substracting $S^{(0)}(k)$ in the integrand of (9), and switching to dimensionless variables $(q=a k)$, we obtain :

$$
\begin{aligned}
\frac{\beta F}{N} & =\frac{\beta F^{(0)}}{N}-\frac{\Gamma}{\pi} \int_{0}^{\infty} S^{(0)}(q) \frac{q_{\mathrm{TF}}^{2}}{q^{2}+q_{\mathrm{TF}}^{2}} \mathrm{~d} q- \\
& -\frac{\Gamma}{\pi} \int_{0}^{1} \mathrm{~d} \lambda\left[S(q ; \lambda)-S^{(0)}(q)\right] \frac{q_{\mathrm{TF}}^{2}}{q^{2}+q_{\mathrm{TF}}^{2}} \mathrm{~d} q
\end{aligned}
$$

If we set $S(q ; \lambda) \equiv S^{(0)}(q)$ for all $\lambda$, the third term in (10) vanishes and the expression for $\frac{F}{N k T}$ reduces to the usual first order perturbation result [6]. Under normal white dwarf conditions

$$
q_{\mathrm{TF}}=\left(\frac{12 Z}{\pi}\right)^{1 / 3} r_{\mathrm{s}}^{1 / 2} \simeq 0.25
$$

a relatively small number. Hence it seems appropriate to expand the first order term in powers of $q_{\mathrm{TF}}$. 
Bearing in mind that $S^{(0)}(q) \simeq q^{2} / 3 \Gamma$ in the limit $q \rightarrow 0[1,10]$, it is readily verified that :

$$
\begin{aligned}
\frac{\beta F^{(1)}}{N}=-\frac{\Gamma}{\pi} \int_{0}^{\infty} S^{(0)}(q) \frac{q_{\mathrm{TF}}^{2}}{q^{2}+q_{\mathrm{TF}}^{2}} \mathrm{~d} q= \\
\quad=-\frac{\Gamma q_{\mathrm{TF}}^{2}}{\pi} I_{1}(\Gamma)+\frac{q_{\mathrm{TF}}^{3}}{6}+0\left(q_{\mathrm{TF}}^{4}\right)
\end{aligned}
$$

where :

$$
I_{1}(\Gamma)=\int_{0}^{\infty} \frac{S^{(0)}(q)}{q^{2}} \mathrm{~d} q
$$

can be calculated using the OCP structure factors [1]. The numerical data for $I_{1}(\Gamma)$ can be fitted over the whole $\Gamma$ - range of the fluid by the simple expression :

$I_{1}(\Gamma)=\frac{1}{\Gamma^{1 / 2}}\left[a_{1}\left(a_{2}+\Gamma\right)^{1 / 2}+a_{3}\left(a_{4}+\Gamma\right)^{-1 / 2}\right]$

with $a_{1}=0.3260702, a_{2}=4.592037, a_{3}=2.413892$, and $a_{4}=134.47101$. This expression has the correct Debye-Hückel limit $\frac{\pi}{2 \sqrt{3}} \Gamma^{-1 / 2}$ when $\Gamma \rightarrow 0$, and decreases towards $a_{1}$ as $\Gamma \rightarrow \infty$. We have checked numerically that the terms of higher order in $q_{\mathrm{TF}}$, which have been neglected in (11), contribute very little for $q_{\mathrm{TF}} \simeq 1 / 4$, and only about $5 \%$ for $q_{\mathrm{TF}} \simeq 1$.

Finally, in order to estimate the contribution of the third term in (10) (which includes all terms beyond first order in the perturbation series), we approximate $S(q ; \lambda)$ by the generalized RPA expression [11] :

$$
\begin{aligned}
S(q ; \lambda)=\frac{S^{(0)}(q)}{1+\lambda \widetilde{w}(q) S^{(0)}(q)}= & \\
= & \frac{S^{(0)}(q)}{1-\lambda \frac{3 \Gamma q_{\mathrm{TF}}^{2} S^{(0)}(q)}{q^{2}\left(q^{2}+q_{\mathrm{TF}}^{2}\right)}} .
\end{aligned}
$$

Using (13), the $\lambda$-integration in (10) is straightforward, yielding :

$$
\begin{aligned}
& \frac{\beta F}{N}=\frac{\beta F^{(0)}}{N}+\frac{\beta F^{(1)}}{N}-\frac{1}{3 \pi} \int_{0}^{\infty} \times \\
& \times\left\{1-\frac{\log \left[1+\widetilde{w}(q) S^{(0)}(q)\right]}{\tilde{w}(q) S^{(0)}(q)}\right\} \tilde{w}(q) S^{(0)}(q) q^{2} \mathrm{~d} q .
\end{aligned}
$$

In the following, the last term in (14) will be denoted by $I_{2}(\Gamma)$. Because $\tilde{w}(q)<0$ and $S^{(0)}(q) \geqslant 0, I_{2}<0$, in agreement with the Gibbs-Bogolioubov inequality $\frac{\beta F}{N} \leqslant \frac{\beta F^{(0)}}{N}+\frac{\beta F^{(1)}}{N}$. Also, for all physically reasonable values of $q_{\mathrm{TF}}$ and $\Gamma, \tilde{w}(q) S^{(0)}(q)>-1$. Expanding $I_{2}$ in powers of $q_{\mathrm{TF}}$, the leading term is found to be :

$$
I_{2}=-\frac{q_{\mathrm{TF}}^{3}}{24}+0\left(q_{\mathrm{TF}}^{4}\right) .
$$

This contribution is exactly $1 / 4$ of the $q_{\mathrm{TF}}^{3}$ contribution to the first order term (11), which in turn is only a small fraction (a few percent) of the leading $q_{\mathrm{TF}}^{2}$ term for $q_{\mathrm{TF}}<1$ and $\Gamma \gtrsim 1$. Hence in this range of $q_{\mathrm{TF}}$ and $\Gamma$ values to have a good approximation, we can write :

$$
\frac{\beta F}{N}=\frac{\beta F^{(0)}}{N}-\frac{\Gamma I_{1}(\Gamma)}{\pi} q_{\mathrm{TF}}^{2}+0\left(q_{\mathrm{TF}}^{3}\right)
$$

and for the equation of state of the ions :

$$
\begin{aligned}
\frac{\beta P V}{N}=- & V \frac{\partial}{\partial V}\left(\frac{\beta F}{N}\right)= \\
& =\frac{\beta P^{(0)} V}{N}+\frac{\Gamma^{2} I_{1}^{\prime}(\Gamma)}{3 \pi} q_{\mathrm{TF}}^{2}+0\left(q_{\mathrm{TF}}^{3}\right) .
\end{aligned}
$$

where $I_{1}^{\prime}(\Gamma)=\frac{\mathrm{d}}{\mathrm{d} \Gamma} I_{1}(\Gamma)$

Eq. (16) and (17) are only valid in the limit of small $q_{\mathrm{TF}}$, e.g. under white dwarf conditions. For $q_{\mathrm{TF}} \simeq 1$, the full expressions (14) and (11) must be calculated numerically, using the $S^{(0)}(q)$ computed in [1].

For $q_{\mathrm{TF}}>1$ the present simple perturbation theory is inadequate for two reasons. First, the generalized RPA expression for $S(q ; \lambda)$ used in (14) is expected to become less and less accurate as $q_{\mathrm{TF}}$, and hence, the perturbation potential $\widetilde{w}(q)$, increases. Second, the simple Thomas-Fermi expression for $\varepsilon(k)$ is a very poor approximation in the limit of strong screening, i.e. large $q_{\mathrm{TF}}$. Finally in this limit the OCP is not expected to be a reasonable reference system, because the effective ion-ion interaction becomes short ranged.

\section{References}

[1] Hansen, J. P., Phys. Rev. A 8 (1973) 3096.

[2] Pollock, E. L. and Hansen, J. P., Phys. Rev. A 8 (1973) 3110.

[3] Hansen, J. P., Mc Donald, I. R. and Pollock, E. L., to appear in Phys. Rev. A (1975).

[4] Hubbard, W. B. and Slattery, W. L., Astrophys. J. 168 (1971) 131.

[5] See, e.g. PInEs, D., Elementary Excitations in Solids (W. A. Benjamin, Inc., New York) 1964.

[6] Zwanzig, R. W., J. Chem. Phys. 22 (1954) 1420.

[7] Ross, M. and Seale, D., Phys. Rev. A 9 (1974) 396.
[8] LindhaRd, J., K. Danske Vidensk. Selsk. Mat-Fys. Medd. 28 (1954) 8.

[9] See e.g. Weeks, J. D., Chandler, D. and Andersen, H. C., J. Chem. Phys. 54 (1971) 5237.

[10] Stillinger, F. H. and Lovett, R., J. Chem. Phys. 49 (1968) 1991.

[11] Broyles, A. A., Sahlin, H. L. and Carley, D. D., Phys. Rev. Lett. 10 (1963) 319;

Lado, F., Phys. Rev. A 135 (1964) 1013. 